\title{
Metarubricyte Count
}

National Cancer Institute

\section{Source}

National Cancer Institute. Metarubricyte Count. NCI Thesaurus. Code C128972.

The determination of the amount of metarubricytes present in a sample. 